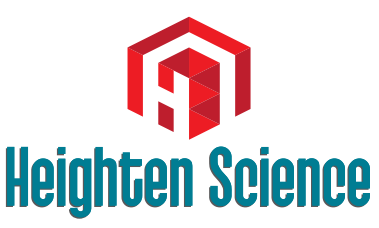

P U B L I C A T I O N S Corporation
*Address for Correspondence: Ellabib M, Department of Medical Microbiology and Immunology, Faculty of Medicine, Tripoli University, P.O. Box 13497, Tripoli, Libya, Tel: 0021-8925 857619; Email: ellabib@mail.com

Submitted: 01 August 2018

Approved: 13 August 2018

Published: 14 August 2018

Copyright: @ 2018 Krema ZA, et al. This is an open access article distributed under the Creative Commons Attribution License, which permits unrestricted use, distribution, and reproduction in any medium, provided the original work is properly cited.

Keywords: Cryptococcus neoformans; Melanin production; Differential medium; Seeds, Leaves

Check for updates
Research Article

\section{Evaluation of novel culture media prepared from plant substrates for isolation and identification of Cryptococcus Neoformans Species Complex}

\author{
Krema ZA', Mokthar ES ${ }^{1}$, El Magrahi HS², Eshwika A ${ }^{3}$, Ellabib \\ $\mathrm{MS}^{1 *}$ and Cogliati $\mathrm{M}^{4}$ \\ 'Department of Medical Microbiology and Immunology, Faculty of Medicine, Tripoli University, \\ P.O. Box 13497, Tripoli, Libya \\ ${ }^{2}$ Department of Medical Laboratory Science, Faculty of Medical Technology, Tripoli, Libya \\ ${ }^{3}$ Department of Microbiology, Faculty of Pharmacy, Zawia University, Libya \\ ${ }^{4}$ Department Biomedical Sciences for Health, University of Milan, Milan, Italy
}

\section{Abstract}

Background: Melanin production due to phenoloxidase activity is a distinctive property of Cryptococcus neoformans and Cryptococcus gattii species complex yeasts. Therefore, an agar medium containing a precursor of melanin pigment is potentially useful to identify and differentiate cryptococcal colonies from other yeasts.

Purpose: This study aimed to evaluate and compare the ability of Cryptococcus neoformans species complex isolates to produce brown-pigmented colonies when grown on media prepared from various plant leaves or seeds extracts.

Materials and Methods: Forty-six C. neoformans species complex isolates which were obtained from various environmental and clinical samples were inoculated on different media containing coriander, cumin, soybean, lupine, flax, pumpkin, basil, peppermint, and marjoram, were observed for the rate of growth and pigment production during a five-day period.

Results: All isolates were pigmented on all media within 24-48 hours, and brown or dark brown colonies were observed in less than five days, while $C$. albicans grew but did not produce any pigment.

Conclusion: The differential media tested in the present study are simple and inexpensive, and represent alternative valid tools for presumptive identification of $C$. neoformans species complex from clinical and environmental samples.

\section{Introduction}

Cryptococcus neoformans species complex is an opportunistic fungal human pathogen found worldwide which causes life-threatening meningoencephalitis in immunocompromised people. The most serious cryptococcal infections develop in patients with AIDS or reticuloendothelial malignancy, organ transplantation and corticosteroid treatment [1]. C. neoformans species complex major virulence factors include the ability to grow at $37^{\circ} \mathrm{C}$, production of a polysaccharide capsule, and melanin formation [2]. Identification of medical important Cryptococcus species such as $C$. neoformans and C. gattii species complexes takes into account particular characteristics 
of this genus in which the majority of yeasts produces a polysaccharide capsule, grow at $37^{\circ} \mathrm{C}$, and produce urease and laccase enzymes [3]. In addition, $C$. neoformans and C. gattii species complex yeasts are able to produce melanin when cultured in media containing phenolic or polyphenolic substrates [4]. The laccase enzyme present in these yeasts utilizes phenolic substrates generating quinones, which undergo a process of autopolimerization that leads to melanin synthesis. The dark pigment retained in the cell wall of the fungus is responsible for the color shown by the colony [5]. Colonies of melanin-producing Cryptococcus species show a range of colors varying from brown to black when grown in agar media such as sunflower seed agar [6], Niger seed agar [7] and caffeic acid agar [8]. Some recent studies have also shown the production of pigment in mustard seed agar [9], henna agar [10], tobacco agar [11], apple leaves agar, and eggplant leaves agar [12].

The present study describes the evaluation of several novel media containing leaves or seeds extract of coriander (Coriandrum sativum), cumin (Cuminum cyminum), soybean (Glycine max), lupine (Lupinus albus), pumpkin (Cucurbita maxima), flax (Linum usitatissimum), basil (Ocimum basilicum), peppermint (Mentha viridis), and marjoram (Origanum majorana). The study was based on the fact that these plant leaves or seeds are rich sources in antioxidant compounds such as phenolic and polyphenolic which supports growth of cryptococcal yeasts as well as production of melanin pigments, and allow their easy differentiation by formation of brown colored colonies. To the best of our knowledge this is the first study using the preparation of these differential medium substrates for $C$. neoformans species complex isolation and differentiation from either clinical or environmental sources.

\section{Materials and Methods}

Seeds of coriander, cumin, soybean, lupine, flax, pumpkin, and leaves of basil, peppermint, marjoram, were obtained from local market and grinded in a domestic blender in order to obtain a fine powder. Fifty grams of each type of substrate were dissolved in $1 \mathrm{~L}$ of distilled water. The mixture was boiled for $30 \mathrm{~min}$ and then cooled and filtered through gauze. Final volume was adjusted to $1 \mathrm{~L}$ with distilled water. The $\mathrm{pH}$ was adjusted to 6.0, and $20 \mathrm{~g}$ agar-agar (Difco Laboratories, Detroit, MI, USA) was added before the mixture was autoclaved at $121^{\circ} \mathrm{C}$ for $15 \mathrm{~min}$. The medium was allowed to cool to $45-55^{\circ} \mathrm{C}$ and dispensed into sterile Petri dishes. Media containing a mixture of soybean $(5 \%)$ and coriander $(2.5 \%)$ seeds extract, and a mixture of lupine $(5 \%)$ and cumin $(2.5 \%)$ seeds extract were also prepared and evaluated for possible synergism for pigment production.

A total of 44 C. neoformans isolates from various environmental samples collected in Libya [13], and two from Libyan HIV-infected patients [14], were included in the present study. In addition, ten samples from pigeon droppings, previously found to be contaminated by $C$. neoformans species complex, were tested on all media. One gram of dried pigeon droppings was added to $9 \mathrm{ml}$ of sterilized saline solution 0.85 $\%$ supplemented with chloramphenicol $(10 \mu \mathrm{g} / \mathrm{ml})$ and ampicillin $(200 \mu \mathrm{g} / \mathrm{ml})$, and each sample was shaken vigorously for $5 \mathrm{~min}$ on a vortex type agitator. The mixture was kept at room temperature for about 10-15 min. Then, a tenfold serial dilution was made and aliquots of $0.1 \mathrm{ml}$ from the supernatant were spread onto duplicate plates of each medium supplemented with antibiotics (as above) and biphenyl (0.1\%). Plates were incubated at both 25 and $37^{\circ} \mathrm{C}$ for $2-7$ days. The pigment production by cryptococcal yeasts was observed on sunflower seed agar as positive control [6]. Two isolates of Candida albicans were tested as negative control. All tested isolates were initially grown on Sabouraud dextrose agar at $37^{\circ} \mathrm{C}$ for 48 h. C. neoformans species complex and $C$. albicans isolates were cultured onto all media and incubated at $37^{\circ} \mathrm{C}$ for five days. Growth and pigment production were checked daily. 


\section{Results}

All 46 C. neoformans specie complex isolates and the two C. albicans isolates showed a good growth after 24 hours post inoculation onto the media prepared with the nine different substrates. All isolates of $C$. neoformans showed brown colonies on all media within 48 hours post inoculation and were easily identified as $C$. neoformans species complex (Table 1 and Figure 1). In contrast, $C$. albicans colonies remained white in all media up to the maximum period of incubation of 5 days (Figure 1). All 44 C. neoformans isolates produced a brown pigment on sunflower seed agar control medium.

When $C$. neoformans species complex isolates were cultured on a medium containing a combination of substrates such as soybean with coriander or lupine with cumin, pigment production was found to be more intense, already within 24-48 h incubation, than that observed in on the plates containing only a single substrate. In addition, growth on these media was not inhibited or delayed (Figure 1).

C. neoformans species complex isolates were easily identified from pigeon dropping samples as brown-pigmented colonies on all media and were easily differentiated from other yeasts (Figure 2).

Table 1: Number of days for growth and pigment formation of Cryptococcus neoformans species complex isolates cultured on different media.

\begin{tabular}{|c|c|c|}
\hline Medium substrate & Growth (days) & Pigment formation (days) \\
\hline Coriander seeds & 1 & 2 \\
\hline Cumin seeds & 1 & 2 \\
\hline Soybean seeds & 1 & 2 \\
\hline Lupine seeds & 1 & 2 \\
\hline Flax seeds & 1 & 2 \\
\hline Pumpkin seeds & 1 & 2 \\
\hline Basil leaves & 1 & 2 \\
\hline Peppermint leaves & 1 & 2 \\
\hline Marjoram leaves & 1 & 2 \\
\hline Soybean plus coriander & 1 & 2 \\
\hline Lupine plus cumin & 1 & 2 \\
\hline Sunflower seeds & 1 & 2 \\
\hline
\end{tabular}

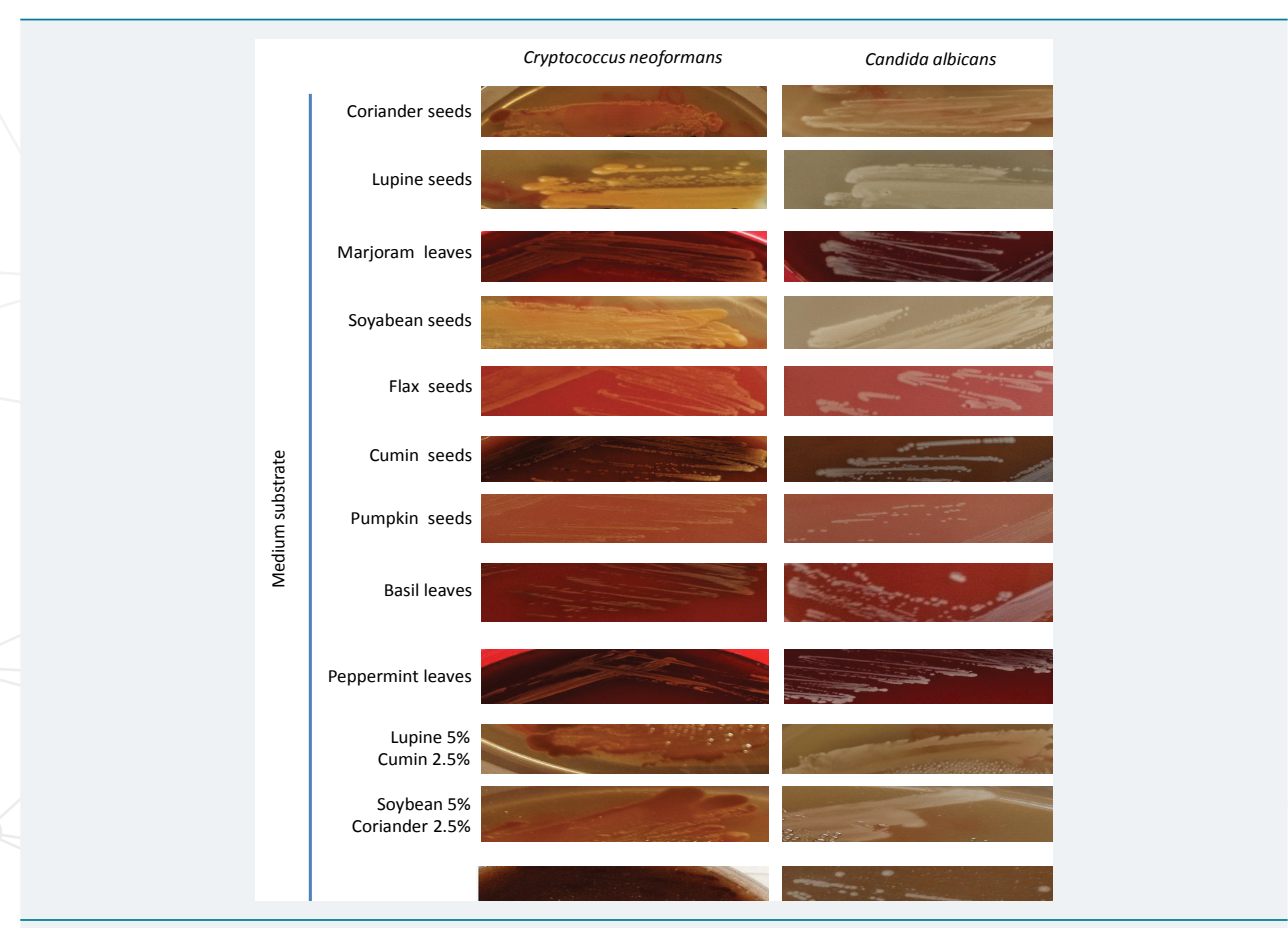

Figure 1: Brown-pigmented colonies of Cryptococcus neoformans species complex grown after 48 hours on media prepared with different plant substrates compared to Candida albicans white colonies. 

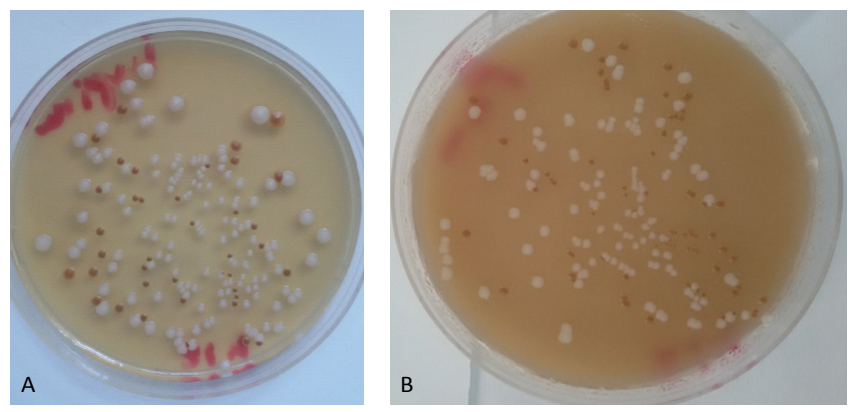

Figure 2: Cryptococcus neoformans species complex isolated from pigeon dropping samples as brown-pigmented colonies and Candida albicans as white colonies. A) Pumpkin seed agar medium; B) lupine seed agar medium

\section{Discussion}

Several studies reported the appearance of brown-pigmented colonies of $C$. neoformans species complex using various culture media [6,7,10-12]. Our study confirms that media containing plant materials, without any supplemental source of carbon, nitrate or vitamin, are able to allow the growth of $C$. neoformans species complex yeasts. This is in agreement with previous studies reporting the ability of $C$. neoformans and C. gattii species complexes to grow and mate on different plant materials such as Arabidospsis thaliana, Colophospermum mopane, Eucalyptus camaldulensis, Pseudotsuga menziesii and other conifers [15]. In addition, our study confirms the presence of phenolic and polyphenolic compounds, which can be assimilated and utilized by cryptococcal yeasts as substrates for melanin synthesis, in all the plant materials employed for media preparation.

A number of selective media for isolation and identification of $C$. neoformans species complex are sold by several companies supplier, however, the main problems are that these media are not available in low-income countries market and they have high costs. The media described in the present study require seeds and leaves which are inexpensive and widely available in the market, and do not require any additive, therefore they are optimal for those laboratories that cannot afford costs to buy commercial media. This study also extends the range of plant substrates useful for cryptococcal yeasts growth revealing the potential ability of these pathogens to colonize most of the plants that contains phenolic and polyphenolic compounds. Finally, we show that production of brown pigment enhanced and synergism observed when two substrates such as soybean plus coriander and lupine plus cumin combined. This synergism and enhancements in pigment production may attributed to the presence of different in phenolic compound in both coriander and cumin which contain more caffeic acid and chlorogenic acid $[16,17]$ which play important role in melanin and pigment production by C. neoformans comlex., while soybean and lupin contain mainly gallic acid, p-Hydroxybenzoic, less caffeic acid, p-coumaric and ferulic acid $[18,19]$. This may explain the intensity of brown pigment color when both soybean and lupin was combined with either coriander or cumin.

In conclusion, we confirm that melanization of $C$. neoformans species complex colonies is an important characteristic for differentiation of this pathogen from other yeasts and that many common plant substrates could be employed for differential media preparation making this approach affordable in any microbiology laboratory.

\section{References}

1. Rajasingham R, Smith RM, Park BJ, Jarvis JN, Govender NP, et al. Global burden of disease of HIVassociated cryptococcal meningitis: an updated analysis. Lancet Infect Dis. 2017; 17: 873-881. Ref.: https://tinyurl.com/y9kkgk8e

2. Heitman J, Kozel TR, Kwon-Chung KJ, Perfect JR, Casadevall A. Cryptococcus: From Human Pathogen to Model Yeast. ASM Press, Washington, DC, USA. 2011. Ref.: https://tinyurl.com/yd53oxge 
3. De Hoog GS, Guarro J, Gene J, Figueras MJ. Atlas of clinical fungi. ASM Press, Washington, DC, USA 2000.

4. Lacaz CS, Porto E, Martins JEC. Micologia media: fungos, actinomicetos ealgas de interesse medico. 8th ed. Sao Paulo: Sarvier. 1991.

5. Chaskes S, Tyndall RL. Pigment production by Cryptococcus neoformans from para and ortho-diphenols: effect of the nitrogen source. J Clin Microbiol. 1975; 1: 509-514. Ref.: https://tinyurl.com/y8us93ja

6. Pal M, Mehrotra BS. Studies on the efficacy of sunflower seed agar for the isolation and identification of Cryptococcus neoformans. Arogya J Health Sci 1982; 8: 74-79.

7. Strachan AA, Yu RJ, Blank F. Pigment production of Cryptococcus neoformans grown with extracts of Guizotia abyssinica. Appl Microbiol. 1971; 22: 478-489.

8. Hopfer RL, Blank F. Caffeic acid-containing medium for identification of Cryptococcus neoformans. J Clin Microbiol. 1976; 2: 115-120. Ref.: https://tinyurl.com/ycwcbdn4

9. Nandhakumar B, Kumar G, Prabhu CP, Menon T. Mustard seed agar,a new medium for the differentiation of Cryptococccus neoformans. J Clin Microbiol. 2006; 44: 674. Ref.: https://tinyurl.com/y78ymenx

10. Nandhakumar B, Menon T, Kumar G. A new henna-based medium for the differentiation of Cryptococcus neoformans. J Med Microbiol. 2007; 56: 568. Ref.: https://tinyurl.com/yba8zu4q

11. Tendolkar U, Tainwala S, Jog S, Mathur M. Use of a new medium - tobacco agar, for pigment production of Cryptococcus neoformans. Indian J Med Microbiol. 2003; 21: 277-279. Ref.: https://tinyurl.com/ybtory4b

12. Ajah HA. Two new media apple leaves agar and eggplant leaves agar for identification of Cryptococcus neoformans. J Biology, Agriculture and Healthcare. 2014; 4. Ref.: https://tinyurl.com/y7al3pva

13. Ellabib MS, Krema ZA, Allafi AA, Cogliati M. First report of two cases of cryptococcosis in Tripoli, Libya, infected with Cryptococcus neoformans isolates present in the urban area. J Mycol Med. 2017; 27: 421-424. Ref.: https://tinyurl.com/ycwn9gb4

14. Ellabib MS, Aboshkiwa MA, Husien WM, D'Amicis R, Cogliati M. Isolation, identification and molecular typing of Cryptococcus neoformans from pigeon droppings and other environmental sources in Tripoli, Libya. Mycopathologia. 2016; 181: 603-608. Ref.: https://tinyurl.com/ycbvydzl

15. Springer DJ, Mohan R, Heitman J. Plants promote mating and dispersal of the human pathogenic fungus Cryptococcus. PLoS One. 2017; 12: e0171695. Ref.: https://tinyurl.com/y8sr4ctv

16. Rajeshwari CU, Andallu B. Reverse phase HPLC for the detection of flavonoids in the ethanolic extract of Coriandrum sativum L seeds. International Journal of Basic and Applied Sciences. 2012; 1: 21-26.

17. Claudia-CT, Neli-Kinga O, Laurian V, Cristina M. Comparative Studies on Polyphenolic Composition, Antioxidant and Diuretic Effects of Nigella sativa L. (Black Cumin) and Nigella damascena L. (Ladyin-a-Mist) Seeds. Molecules. 2015; 20: 9560-9574. Ref.: https://tinyurl.com/y88sgcq9

18. Montserrat D, Teresa H, Sergio R, Grzegorz L, Isabel E, et al. Bioactive Phenolic Compounds of Soybean (Glycine max cv. Merit): Modifications by Different Microbiological Fermentations. Pol J Food Nutr Sci. 2012; 62: 241-250. Ref.: https://tinyurl.com/ya8azlua

19. Aleksander S, Jaroslaw C, Piotr K, Krzysztof D, Eleonora LS, et al . Antioxidant activity and phenolic content in three lupin species. Journal of Food Composition and Analysis. 2012; 25: 190-197. Ref.: https://tinyurl.com/y7fvumzg 